\title{
Serum asymmetric dimethylarginine and nitric oxide levels in Turkish patients with acute ischemic stroke
}

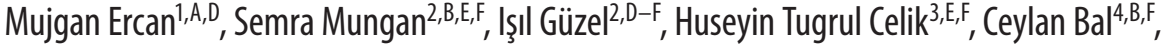 \\ Sedat Abusoglu ${ }^{5, C, F}$, Deniz Akbulut ${ }^{6, D, F}$, Esra Firat Oguz ${ }^{7, A, F}$, Fatma Meric Yilmaz ${ }^{8, C, F}$ \\ ${ }^{1}$ Department of Biochemistry, Aydın Public Health Laboratory, Turkey \\ ${ }^{2}$ Department of Neurology, Ankara Numune Training and Education Hospital, Turkey \\ ${ }^{3}$ Department of Biochemistry, Faculty of Medicine, Turgut Ozal University, Ankara, Turkey \\ ${ }^{4}$ Department of Biochemistry, $4^{\text {th }}$ Ankara Occupational Diseases Hospital, Turkey \\ ${ }^{5}$ Department of Biochemistry, Faculty of Medicine, Selçuk University, Konya, Turkey \\ ${ }^{6}$ Çukurova Dr. Aşkım Tüfekçi Hospital, Adana, Turkey \\ ${ }^{7}$ Department of Biochemistry, Ankara Numune Training and Education Hospital, Turkey \\ ${ }^{8}$ Department of Biochemistry, Faculty of Medicine, Ylldırım Beyazit University, Ankara, Turkey \\ A - research concept and design; $B$ - collection and/or assembly of data; $C$ - data analysis and interpretation; \\ $D$ - writing the article; $E$ - critical revision of the article; $F$ - final approval of the article
}

\section{Address for correspondence}

|şıl Güzel

E-mail: dr.dulda@hotmail.com

Funding sources

None declared

Conflict of interest

None declared

Acknowledgements

We are grateful to Roche and Beckman Diagnostics for providing reagent kits free of charge for this study.

Received on 0ctober 28, 2015

Reviewed on March 3, 2016

Accepted on 0ctober 6, 2017

Published online on August 29, 2018

Cite as

Ercan M, Mungan S, Güzel I, et al. Serum asymmetric dimethylarginine and nitric oxide levels in Turkish patients with acute ischemic stroke. Adv Clin Exp Med. 2019;28(5):693-698. doi:10.17219/acem/78360

D0I

$10.17219 /$ acem/78360

\section{Copyright}

Copyright by Author(s)

This is an article distributed under the terms of the

Creative Commons Attribution Non-Commercial License

(http://creativecommons.org/licenses/by-nc-nd/4.0/)

\section{Abstract}

Background. Nitric oxide synthase (NOS) is present in the brain and cerebral arteries and it enables the synthesis of nitric oxide (NO), which plays a critical role in brain perfusion. Asymmetrical dimethylarginine (ADMA) is an endogenous NOS inhibitor.

Objectives. The aim of this study was to evaluate serum ADMA levels, which are an indicator of endothelial dysfunction of the renal functions in patients with acute ischemic stroke, and to determine whether there is a possible correlation between ADMA and NO levels and the L-arginine-to-ADMA ratio.

Material and methods. Fifty-two patients (22 male and 30 female; mean age: $75.2 \pm 10.1$ years) with a diagnosis of acute ischemic stroke in the first $24 \mathrm{~h}$ post-stroke and 48 healthy individuals (controls; 13 male and 35 female; mean age: $60.1 \pm 7.92$ years) were included in this study. The risk factors recorded and evaluated were age and gender of the patients, serum lipid levels, serum ADMA levels, nitrate-to-nitrite ratios, L-arginine, L-arginine-to-ADMA ratios, sedimentation rate, (-reactive protein (CRP), urea and creatinine levels, and glomerular filtration ratio (eGFR).

Results. The mean serum ADMA level was $0.48 \pm 0.23 \mu \mathrm{M}$ for the patients and $0.36 \pm 0.18 \mu \mathrm{M}$ for the controls. The mean N0 level was $2.78 \pm 0.59 \mu \mathrm{M}$ for the patient group and $4.49 \pm 2.84 \mu \mathrm{M}$ for the controls. The ADMA levels for the patient group were significantly higher than for the control group $(p=0.011)$; the NO levels for the patients were significantly lower than for the controls $(p<0.001)$. The logistic regression method demonstrated that ADMA and NO levels may be independent risk factors for the patient group, and the receiver operating characteristic (ROC) curve analysis showed that both of these variables were discriminative risk factors.

Conclusions. An increased serum level of the NOS inhibitor ADMA was found to be a possible independent risk factor for ischemic stroke.

Key words: nitric oxide, asymmetrical dimethylarginine, asymmetrical dimethylarginine/arginine levels, arginine, acute ischemic stroke 


\section{Introduction}

Nitric oxide synthase (NOS), a requirement for the synthesis of nitric oxide (NO), which plays a critical role in the control of brain perfusion, is located in the brain and the walls of the cerebral arteries. ${ }^{1-3}$ Asymmetrical dimethylarginine (ADMA) is a competitive endogenous inhibitor of endothelial NOS, which decreases endothelial $\mathrm{NO}$ synthesis and leads to the loss of NO bioavailability. ${ }^{3}$ Age, diabetes mellitus (DM), hypertension (HT), carotid arterial intima-media thickness, hyperlipidemia, hyperhomocysteinemia, obesity, inflammation, and sickle cell disease have all been found to be associated with increased ADMA blood levels. ${ }^{1,4}$ In addition, ADMA has been suggested to be an independent marker of acute stroke and transient ischemic attack. ${ }^{5-7}$

Increased ADMA concentration has been found to be associated with a more rapid renal disease progression and mortality via reducing NO bioavailability, which results in inflammation and oxidative stress - typical features of renal disease progression. ${ }^{8-10}$ Asymmetrical dimethylarginine is also considered to be a uremic toxin, so it is also possible that ADMA contributes to progressive renal dysfunction by functionally impairing the integrity of the glomerular filtration barrier, promoting proteinuria, interstitial and glomerular fibrosis, and oxidative stress. ${ }^{11,12}$ Although ADMA is attributed to impaired renal function, it is not yet clear whether ADMA is a marker or a risk factor for renal disease progression.

In this study, we aimed to investigate the relationship between serum levels of ADMA and NO and the L-arginineto-ADMA ratio (indicators of endothelial dysfunction) in patients with acute ischemic stroke, and to discuss the possible confounding effect of renal function on ADMA and NO levels.

\section{Material and methods}

\section{Ethics statement}

This study was approved by the Ethical Committee of Ankara Numune Training and Research Hospital, Turkey. Informed consent was obtained from each participant before the study.

\section{Study population}

A total of 52 patients who were admitted to the Department of Neurology of Ankara Numune Training and Research Hospital, Turkey, between December 2009 and May 2010 with a diagnosis of acute ischemic stroke in the first $24 \mathrm{~h}$ post-stroke and 48 healthy individuals were included in the study.

The diagnosis of ischemic stroke was based on the patient's history, clinical examination, computed tomography (CT) examination, and magnetic resonance imaging (MRI). Hypertension was defined according to the current World Health Organization (WHO) criteria and/or the use of antihypertensive drugs. Stroke severity on admission was assessed using the National Institutes of Health Stroke Scale/ Score (NIHSS). An NIHSS of $0-1$ was considered mild, 2-8 was moderate, and $\geq 9$ was severe. ${ }^{13}$ Patients with hemorrhagic stroke, coronary artery disease, mitral fibrillation, kidney or liver disease, rheumatological or collagenous disease, those with a diagnosis of cancer, fever or infection, those receiving anticoagulation treatment, and those who had undergone vascular surgery in the previous 3 months were excluded from this study.

\section{Methods}

Blood samples were drawn from the patients on the morning of the $1^{\text {st }}$ day of admission to the Department of Neurology into $10 \mathrm{~mm}$ tubes with red caps, not containing gel (BD Vacutainer; Becton, Dickinson and Co., Franklin Lakes, USA). After at least $30 \mathrm{~min}$ of incubation, the specimen was centrifuged at 1,500 $\times \mathrm{g}$ for $10 \mathrm{~min}$, and then lipid profiles and urea, creatinine and $\mathrm{C}$-reactive protein (CRP) levels were determined using commercial kits (Beckman Coulter DXC 800; Beckman Coulter, Inc., Brea, USA). A fibrinogen test was performed using a tube containing citrate and a Siemens CA 7000 device (Siemens Healthcare Diagnostics, Marburg, Germany), and the erythrocyte sedimentation rate (ESR) was determined using a tube containing citrate and a Berkhun SDM 100 device (Blood Testing Equipments; Mediko Dardanel, Canakkale, Turkey). The serum samples to be used in determining ADMA, NO and arginine levels were stored at $-80^{\circ} \mathrm{C}$ until analysis.

Serum ADMA and arginine levels were measured on the same day, after all the samples were collected by using an Applied Biosystems MDS SCIEX API 3200 LC-MS/MS system device (Applied Biosystems, Concord, Canada) in the ESI-positive mode and an Agilent Eclipse XDB-C18 column (Agilent Technologies, Palo Alto, USA). According to this method, the intra-day coefficient of variation $(\mathrm{CV})$ and inter-day CV were $3.9 \%$ and $6.2 \%$, respectively. Nitric oxide level was determined using a Cayman (780001) Nitrate/ Nitrite colorimetric assay kit (Cayman Chemical Company, Ann Arbor, USA). Briefly, the first step is the conversion of nitrate to nitrite-utilizing nitrate reductase. The next step is the addition of Griess reagent, which converts nitrite into a deep purple azo compound. The photometric measurement of the absorbance due to this chromophore accurately determines the $\mathrm{NO}_{2}$ concentration. The intra-assay $\mathrm{CV}$ was $3.4 \%$ and the inter-assay $\mathrm{CV}$ was $2.7 \%$.

\section{Statistical analysis}

The data from the study was analyzed with SPSS software, v. 18.0 (SPSS, Inc., Chicago, USA). The conformity of continuous variables to a normal distribution was 
tested with the Shapiro-Wilk test. The descriptive statistics of continuous variables were expressed as mean \pm standard deviation (SD). The presence of a statistically significant differences between the groups in terms of continuous variables was examined with Student's t-test for parametric variables and with the Mann-Whitney U test for nonparametric variables. The analysis of variance (ANOVA) was used to analyze the differences among group means and their associated procedures. The presence of a correlation among the groups was assessed with Pearson's and Spearman's rho tests. The logistic regression method was performed to demonstrate whether ADMA and NO may be risk factors for stroke and the receiver operating characteristic (ROC) curve analysis was done to check whether these parameters may be discriminative factors for stroke.

\section{Results}

Fifty-two patients with acute ischemic stroke were included in this study; 22 were males and 30 were females. The mean age of the patients was $75.27 \pm 10.05$ years. Thirty-seven patients had HT, 14 had DM and 13 had atrial fibrillation.

The ADMA levels were significantly higher in the patient group than in the control group $(0.46 \pm 0.13 \mu \mathrm{M}$ vs $0.40 \pm 0.11 \mu \mathrm{M}$, respectively) and the NO levels were significantly lower in the patient group than in the controls ( $2.78 \pm 1.59$ vs $4.34 \pm 2.70$, respectively) ( $\mathrm{p}<0.05)$.

There was no statistically significant difference between the serum L-arginine-to-ADMA ratio in the patient group and in the control group ( $\mathrm{p}=0.494)$. The ADMA, CRP, urea, creatinine, and the glomerular filtration ratio (eGFR) levels, and ESR were statistically significantly higher in the patient group than in the controls $(\mathrm{p}<0.05)$. The levels of NO, high-density lipoprotein (HDL)-cholesterol and triglycerides were statistically significantly lower in the patient group than in the controls $(p<0.05)$. There was no statistically significant difference between the L-arginine, total cholesterol, low-density lipoprotein (LDL)-cholesterol, or fibrinogen levels, or the L-arginineto-ADMA ratio in patients compared to the control group $(\mathrm{p}>0.05)$ (Table 1).

There was no statistically significant difference in the ADMA, NO or L-arginine levels, or the L-arginine-toADMA ratio among the different stroke subgroups (mild, moderate or severe, as determined by NIHSS) (Table 2).

There was a negative correlation between the NO levels and age $(r=-0.251)$, and a positive correlation between the NO levels and eGFR ( $r=0.223)$ (Fig. 1). In addition, there was a positive correlation between ADMA and ESR $(r=0.202)$, and between ADMA and creatinine $(r=0.224)$, and there was a negative correlation between ADMA and eGFR $(r=-0.216)$ : the $95 \%$ confidence interval (CI) eGFR lower and upper values were 77.81 and 90.0, respectively (Fig. 1).

According to the ROC curve analysis (Fig. 2), the NO and ADMA levels were found to be discriminative parameters in the patient group. According to the model, serum NO and ADMA levels may be risk factors for the patient group: the odds ratios (OR) and 95\% CI of the risk factors were 1.58 (1.189-2.098) and 1.26 (0.943-1.875), respectively.

Table 1. Demographic characteristics and biochemistry parameters of patients and controls

\begin{tabular}{|c|c|c|c|}
\hline Parameters & Controls $(n=48)$ & Patients $(n=52)$ & $\mathrm{p}$-value \\
\hline Age [years] & $60.10 \pm 7.92$ & $75.20 \pm 10.10$ & $<0.05$ \\
\hline Gender (M/F) & $13 / 35$ & $22 / 30$ & 0.610 \\
\hline ADMA $[\mu \mathrm{M}]$ & $0.40 \pm 0.11$ & $0.46 \pm 0.13$ & 0.005 \\
\hline Nitrate-to-nitrite ratio $[\mu \mathrm{M}]$ & $4.34 \pm 2.70$ & $2.78 \pm 1.59$ & 0.000 \\
\hline L-arginine $[\mu \mathrm{M}]$ & $67.40 \pm 5.90$ & $69.30 \pm 8.65$ & 0.529 \\
\hline L-arginine-to-ADMA ratio & $201.45 \pm 63.4$ & $220.50 \pm 72.30$ & 0.681 \\
\hline Total cholesterol [mg/dL] & $189.10 \pm 37.41$ & $184.15 \pm 45.99$ & 0.576 \\
\hline HDL-cholesterol [mg/dL] & $43.21 \pm 10.91$ & $37.32 \pm 10.82$ & 0.010 \\
\hline LDL-cholesterol [mg/dL] & $117.81 \pm 28.60$ & $120.43 \pm 38.25$ & 0.714 \\
\hline Triglycerides [mg/dL] & $164.57 \pm 117.97$ & $119.10 \pm 68.45$ & 0.022 \\
\hline $\mathrm{ESR}[\mathrm{mm} / \mathrm{h}]$ & $15.55 \pm 10.84$ & $28.31 \pm 20.36$ & 0.000 \\
\hline CRP $[\mathrm{mg} / \mathrm{dL}]$ & $0.54 \pm 0.14$ & $1.66 \pm 2.64$ & 0.008 \\
\hline Fibrinogen $[\mathrm{mg} / \mathrm{dL}]$ & $350.81 \pm 53.80$ & $371.13 \pm 93.85$ & 0.319 \\
\hline Urea [mg/dL] & $25.57 \pm 7.29$ & $39.77 \pm 22.80$ & 0.000 \\
\hline Creatinine $[\mathrm{mg} / \mathrm{dL}]$ & $0.66 \pm 0.16$ & $1.03 \pm 0.52$ & 0.000 \\
\hline eGFR $\left[\mathrm{mL} / \mathrm{min} / 1.73 \mathrm{~m}^{2}\right]$ & $100.30 \pm 24.04$ & $71.66 \pm 30.00$ & 0.000 \\
\hline
\end{tabular}

ADMA - asymmetrical dimethylarginine; CRP - C-reactive protein; eGFR - glomerular filtration ratio; ESR - erythrocyte sedimentation rate; HDL - highdensity lipoprotein; LDL - low-density lipoprotein; $\mathrm{NO}$ - nitric oxide; nitrate-to-nitrite ratio is a measure of NO levels. 
Table 2. ADMA, NO, L-arginine, and L-arginine-to-ADMA ratios in subgroups of stroke according to NIHSS scores

\begin{tabular}{|l|c|c|c|c|}
\multicolumn{1}{|c|}{ Parameters } & $\begin{array}{c}\text { Control } \\
(\mathrm{n}=48)\end{array}$ & $\begin{array}{c}\text { Mild stroke } \\
(\mathrm{n}=3)\end{array}$ & $\begin{array}{c}\text { Moderate stroke } \\
(\mathrm{n}=40)\end{array}$ & $\begin{array}{c}\text { Severe stroke } \\
(\mathrm{n}=9)\end{array}$ \\
\hline ADMA $[\mu \mathrm{M}]$ & $0.40 \pm 0.11$ & $0.45 \pm 0.06$ & $0.46 \pm 0.14$ & $0.50 \pm 0.14$ \\
\hline Nitrate-to-nitrite ratio $[\mu \mathrm{M}]$ & $4.34 \pm 2.70$ & $2.09 \pm 0.16$ & $2.92 \pm 1.71$ & 0.329 \\
\hline L-arginine $[\mu \mathrm{M}]$ & $67.40 \pm 5.90$ & $65.3 \pm 1.81$ & $69.60 \pm 6.55$ & 0.732 \\
\hline L-arginine-to-ADMA ratio & $201.45 \pm 63.40$ & $147.66 \pm 23.35$ & $241.80 \pm 74.60$ & $150.50 \pm 45.00$ \\
\hline
\end{tabular}

ADMA - asymmetrical dimethylarginine; NIHSS - National Institutes of Health Stroke Scale/Score; NO - nitric oxide; nitrate-to-nitrite ratio is a measure of NO levels.
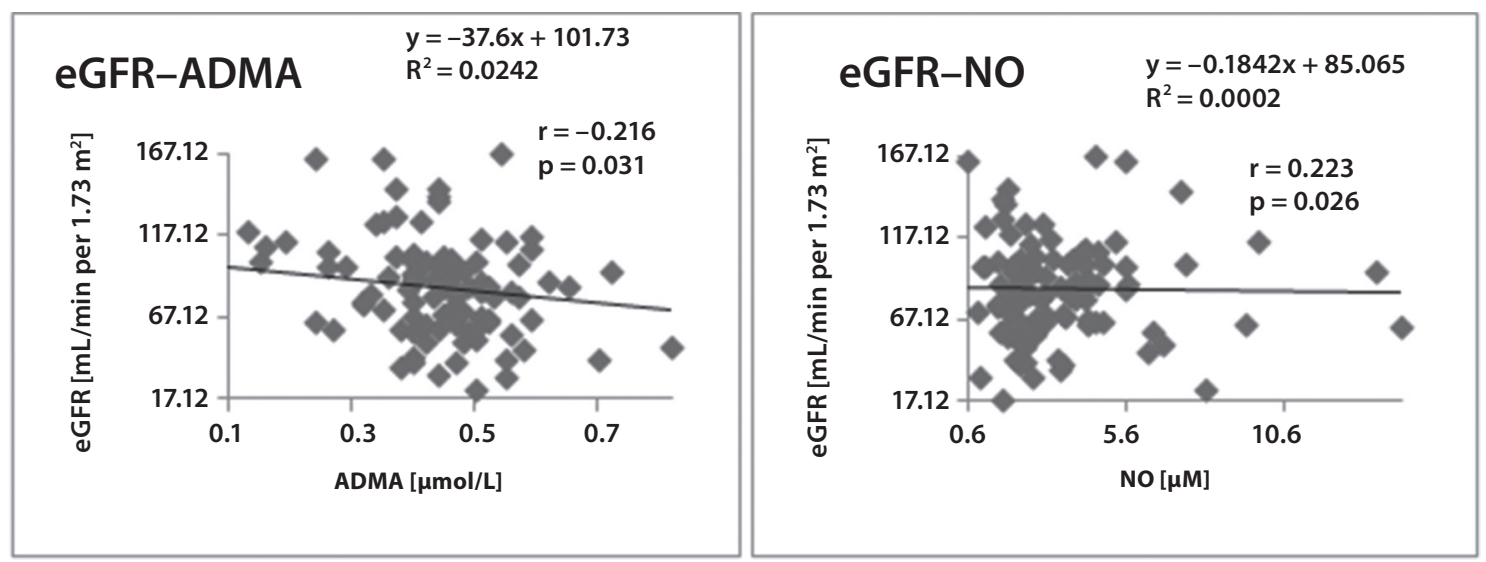

Fig. 1. eGFR-ADMA correlation (left), eGFR-NO correlation (right)

ADMA - asymmetrical dimethylarginine; eGFR - glomerular filtration ratio; NO - nitric oxide.

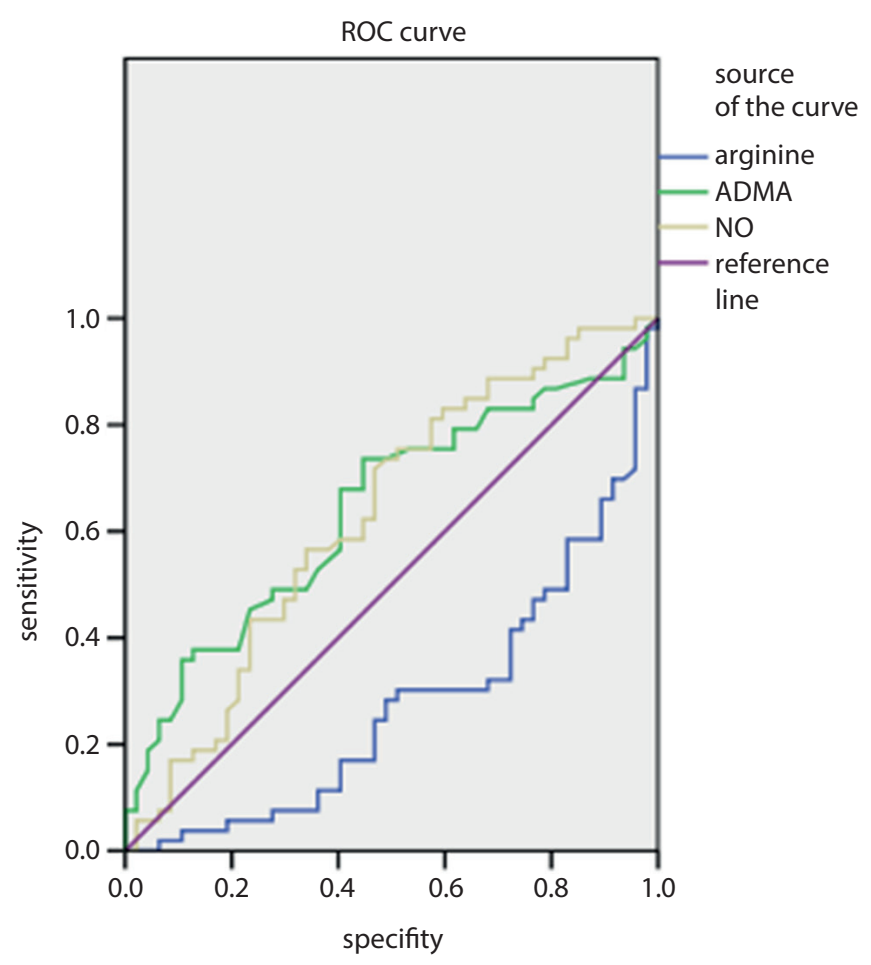

Fig. 2. ROC curve analyzing the discriminative parameters in patients with acute ischemic stroke

ADMA - asymmetrical dimethylarginine; $\mathrm{NO}$ - nitric oxide; $\mathrm{ROC}$ - receiver operating characteristic. Diagonal segments are produced by ties.

\section{Discussion}

Risk factors for stroke, such as HT, DM, smoking, hyperlipidemia, and hyperhomocysteinemia, are associated with endothelial dysfunction. The inhibition of endothelial NO plays an important role in the athero-thrombotic process. ${ }^{14}$ Asymmetrical dimethylarginine, a post-translationally modified form of arginine, ${ }^{15}$ is an endogenous inhibitor of NO synthase and is associated with atherosclerotic diseases. ${ }^{14}$

In this study, serum ADMA levels were increased and NO levels were decreased in patients who had suffered from an acute ischemic stroke as compared to controls. An increased serum ADMA level was determined to be an independent risk factor for ischemic stroke.

Although studies have examined the relationship between ADMA levels and coronary heart disease (CHD), $\mathrm{HT}$, chronic renal failure, and hypercholesterolemia, studies that have investigated the relationship between ADMA levels and stroke are scarce. Yoo and Lee found that serum ADMA levels were significantly higher in 52 patients with stroke than in 36 healthy controls. ${ }^{7}$ In our study, with a similar sample size to Yoo and Lee's study population, ADMA levels were also significantly higher in the patients with acute ischemic stroke than in the controls $(\mathrm{p}=0.005)$.

Prospective clinical studies support the hypothesis that plasma ADMA concentration increases with ischemic 
stroke risk factors and in patients with ischemic stroke. Mamatha et al. found significantly higher serum ADMA levels in stroke patients than in controls in a study that included 201 stroke patients and 217 controls $(\mathrm{p}<0.001) .{ }^{16}$ Increased plasma concentrations of ADMA have been reported to be an independent risk factor for ischemic stroke, after correcting for ischemic stroke risk factors (age, alcohol abuse, smoking, HT, DM, low serum HDL-cholesterol and homocysteine). ${ }^{5}$ In our study, there was no correlation between ADMA and ischemic stroke risk factors (age, DM, HT, serum cholesterol, and serum triglycerides). In a similar study, Nishiyama et al. found that serum ADMA concentrations were significantly higher in 50 stroke patients and 116 individuals with vascular risk factors than in controls with no vascular risk factors. ${ }^{5}$ Asymmetrical dimethylarginine concentrations were found to be associated with vascular risk factors and were suggested as a marker of a future ischemic stroke. ${ }^{16}$

Elevated ADMA levels have been demonstrated in some clinical conditions and in the presence of conventional cardiovascular risk factors, such as high blood pressure, serum cholesterol levels, serum triglycerides, gestational DM, insulin resistance, and smoking. ${ }^{16-21}$ Furthermore, an association between ADMA and non-conventional cardiovascular risk factors, such as elevated levels of homocysteine, CRP, and the vascular cell adhesion molecule (VCAM) has been demonstrated. ${ }^{22-24}$

Acutely decreased NO levels result in vasoconstriction, an increased production of free radicals, platelet aggregation, and leukocyte adhesion on endothelial surfaces; these processes may in turn aggravate cerebral ischemia. ${ }^{25}$ This finding suggests a mechanism for the pathogenesis of ischemic stroke.

In our study, we found significantly lower NO levels in the stroke patients compared to the controls $(\mathrm{p}=0.000)$, suggesting cerebral ischemia due to acute ischemic stroke. Cerebrospinal fluid (CSF) ADMA levels were found to increase in parallel with stroke severity in a study by Brouns et al. ${ }^{25}$ In our study, serum ADMA levels seemed to be higher in patients with more severe strokes; however, the association was not statistically significant $(\mathrm{p}=0.329)$.

A recent study has suggested that renal function may have prognostic value for long-term survival in stroke patients and for the occurrence of cardiovascular events after an acute cerebral event. ${ }^{26}$ In our study, a positive correlation was found between ADMA and creatinine levels $(r=0.224)$, and a negative correlation was observed between ADMA and eGFR $(r=-0.216)$. Some methylarginines are excreted by the renal route. All symmetrical dimethylarginine (SDMA) is excreted by the renal route, whereas ADMA and $\mathrm{N}^{G}$-monomethyl-L-arginine (L-NMMA) are metabolized extensively. The most important step in ADMA metabolism is its breakdown into citrulline and dimethylamine by dimethylarginine dimethylaminohydrolase (DDAH). The lack of SDMA measurements is another limitation of this study.
In conclusion, increased serum levels of the NOS inhibitor ADMA and decreased levels of NO may be independent risk factors for ischemic stroke. The small sample size in the stroke subgroups may be another limitation of this study; further studies with more participants should strengthen the findings of the study. Decreased NO levels cause vasoconstriction and may be important in the pathogenesis of ischemic stroke. Additional comprehensive studies are needed to validate ADMA and NO as routine risk factors of stroke.

\section{References}

1. Kielstein JT, Donnerstag F, Gasper S, et al. ADMA increases arterial stiffness and decreases cerebral blood flow in humans. Stroke. 2006; 37:2024-2029.

2. Landmesser $\mathrm{U}$, Drexler $\mathrm{H}$. The clinical significance of endothelial dysfunction. Curr Opin Cardiol. 2005;20:547-551.

3. Leiper J, Vallance P. Biological significance of endogenous methylarginines that inhibit nitric oxide synthases. Cardiovasc Res. 1999;43: 542-548.

4. Kielstein JT, Zoccali C. Asymmetric dimethylarginine: A cardiovascular risk factor and a uremic toxin coming of age? Am J Kidney Dis. 2005;46:186-202.

5. Nishiyama $Y$, Ueda $M$, Otsuka $T$, et al. Statin treatment decreased serum asymmetric dimethylarginine (ADMA) levels in ischemic stroke patients. J Atheroscler Thromb. 2011;18:131-137.

6. Wanby P, Teerlink T, Brudin L, et al. Asymmetric dimethylarginine (ADMA) as a risk marker for stroke and TIA in a Swedish population. Atherosclerosis. 2006;185:271-277.

7. Yoo JH, Lee SC. Elevated levels of plasma homocyst(e)ine and asymmetric dimethylarginine in elderly patients with stroke. Atherosclerosis. 2001;158:425-430.

8. Alpoim PN, Sousa LP, Mota AP, Rios DR, Dusse LM. Asymmetric dimethylarginine (ADMA) in cardiovascular and renal disease. Clin Chim Acta. 2015;440C:36-39.

9. Raptis V, Kapoulas S, Grekas D. Role of asymmetrical dimethylarginine in the progression of renal disease. Nephrology (Carlton). 2013;18:11-21.

10. Baylis $C$. Nitric oxide synthase derangements and hypertension in kidney disease. Curr Opin Nephrol Hypertens. 2012;21:1-6.

11. Eiselt J, Rajdl D, Racek J, Vostry M, Rulcova K, Wirth J. Asymmetric dimethylarginine and progression of chronic kidney disease: A oneyear follow-up study. Kidney Blood Press Res. 2014;39:50-57.

12. Brunet $P$, Gondouin $B$, Duval-Sabatier $A$, et al. Does uremia cause vascular dysfunction? Kidney Blood Press Res. 2011;34:284-290.

13. The National Institute of Neurological Disorders and Stroke rt-PA Stroke Study Group. Tissue plasminogen activator for acute ischemic stroke. N Engl J Med. 1995;333:1581-1587.

14. Bonetti PO, Lerman LO, Lerman A. Endothelial dysfunction: A marker of atherosclerotic risk. Arterioscler Thromb Vasc Biol. 2003;23:168-175.

15. Boger $\mathrm{RH}$, Vallance $\mathrm{P}$, Cooke JP. Asymmetric dimethylarginine (ADMA): A key regulator of nitric oxide synthase. Atheroscler Suppl. 2003;4:1-3.

16. Mamatha SN, Nagaraja D, Philip M, Christopher R. Asymmetric dimethylarginine as a risk marker for early-onset ischemic stroke in Indian population. Clin Chim Acta. 2011;412:139-142.

17. Achan V, Broadhead M, Malaki M, et al. Asymmetric dimethylarginine causes hypertension and cardiac dysfunction in humans and is actively metabolized by dimethylarginine dimethylaminohydrolase. Arterioscler Thromb Vasc Biol. 2003;23:1455-1459.

18. Kielstein JT, Bode-Boger SM, Frolich JC, Ritz E, Haller H, Fliser D. Asymmetric dimethylarginine, blood pressure, and renal perfusion in elderly subjects. Circulation. 2003;107:1891-1895.

19. Lundman P, Eriksson MJ, Stuhlinger M, Cooke JP, Hamsten A, Tornvall P. Mild-to-moderate hypertriglyceridemia in young men is associated with endothelial dysfunction and increased plasma concentrations of asymmetric dimethylarginine. J Am Coll Cardiol. 2001;38:111-116.

20. Mittermayer F, Mayer BX, Meyer A, et al. Circulating concentrations of asymmetrical dimethyl-L-arginine are increased in women with previous gestational diabetes. Diabetologia. 2002;45:1372-1378. 
21. Stuhlinger MC, Abbasi F, Chu JW, et al. Relationship between insulin resistance and an endogenous nitric oxide synthase inhibitor. JAMA. 2002;287:1420-1426.

22. Boger RH, Bode-Boger SM, Sydow K, Heistad DD, Lentz SR. Plasma concentration of asymmetric dimethylarginine, an endogenous inhibitor of nitric oxide synthase, is elevated in monkeys with hyperhomocyst(e) inemia or hypercholesterolemia. Arterioscler Thromb Vasc Biol. 2000;20: 1557-1564.

23. Zoccali C, Benedetto FA, Maas R, et al. Asymmetric dimethylarginine, $\mathrm{C}$-reactive protein, and carotid intima-media thickness in end-stage renal disease. J Am Soc Nephrol. 2002;13:490-496.
24. Nanayakkara PW, Teerlink T, Stehouwer CD, et al. Plasma asymmetric dimethylarginine (ADMA) concentration is independently associated with carotid intima-media thickness and plasma soluble vascular cell adhesion molecule-1 (sVCAM-1) concentration in patients with mild-to-moderate renal failure. Kidney Int. 2005;68:2230-2236.

25. Brouns R, Marescau B, Possemiers I, Sheorajpanday R, De Deyn PP. Dimethylarginine levels in cerebrospinal fluid of hyperacute ischemic stroke patients are associated with stroke severity. Neurochem Res. 2009;34:1642-1649.

26. Luneburg N, von Holten RA, Topper RF, Schwedhelm E, Maas R, Boger RH. Symmetric dimethylarginine is a marker of detrimental outcome in the acute phase after ischaemic stroke: Role of renal function. Clin Sci (Lond). 2012;122:105-111. 